\title{
Crowding, molecular volume and plasticity: An assessment involving crystallography, NMR and simulations
}

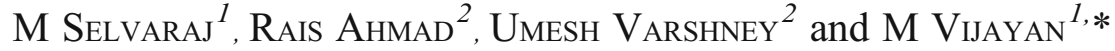 \\ ${ }^{1}$ Molecular Biophysics Unit and ${ }^{2}$ Department of Microbiology and Cell Biology, \\ Indian Institute of Science, Bangalore 560012 \\ *Corresponding author (Fax, +91-80-2360-0535, +91-80-2360-0683; Email, \\ mv@mbu.iisc.ernet.in)
}

\begin{abstract}
The discrepancy between the X-ray and NMR structures of Mycobacterium tuberculosis peptidyl-tRNA hydrolase in relation to the functionally important plasticity of the molecule led to molecular dynamics simulations. The X-ray and the NMR studies along with the simulations indicated an inverse correlation between crowding and molecular volume. A detailed comparison of proteins for which X-ray and the NMR structures appears to confirm this correlation. In consonance with the reported results of the investigations in cellular compartments and aqueous solution, the comparison indicates that the crowding results in compaction of the molecule as well as change in its shape, which could specifically involve regions of the molecule important in function. Crowding could thus influence the action of proteins through modulation of the functionally important plasticity of the molecule.
\end{abstract}

[Selvaraj M, Ahmad R, Varshney U and Vijayan M 2012 Crowding, molecular volume and plasticity: An assessment involving crystallography, NMR and simulations. J. Biosci. 37 1-11] DOI 10.1007/s12038-012-9276-5

\section{Introduction}

The effect of macromolecular crowding on the shape, folding and action of proteins has received considerable attention in recent years (Ellis and Minton 2003; Zhou et al. 2008; Gershenson and Gierasch 2011; Pernilla 2011). This issue is of substantial biological significance as close to half the volume of a typical cell is occupied by biomolecules, and therefore macromolecules like proteins function in a crowded environment in biological systems (Ellis and Minton 2003). This situation is not factored into most of the solution and computational studies on the structure and function of proteins, although there have been a couple of attempts to do so (Ai et al. 2006; Roque et al. 2007; Homouz et al. 2009). Perhaps the only extensive studies which are carried out in an overcrowded environment involve those pursued in crystals where typically the protein molecules occupy $50 \%$ of the volume. Admittedly, in crystals a protein molecule is usually surrounded by molecules of the same kind while the environment is much more heterogeneous inside the cell. Furthermore, the mobility of molecules in crystals is much more limited than in the cell. However, the level of overcrowding is comparable in the two cases. It has also been demonstrated that crystal contacts are different in nature from interactions at interfaces involved in specific assemblies Bahadur et al. (2003)). Therefore, the situation in protein crystals provides a handle, imperfect though it might be, for approaching the problem. The differences in protein structures derived using crystallography and NMR are particularly interesting in this context. Such differences observed in eubacterial peptidyl-tRNA hydrolase are the genesis of the work reported here.

Peptidyl-tRNA hydrolase (Pth), which catalyses the hydrolysis of stalled peptidyl-tRNA during protein synthesis, is an essential enzyme in eubacteria. Premature stalling of translation, caused by a variety of events, leads to the dropping off of peptidyl-tRNA from the ribosome. Accumulation of peptidyl-tRNA is toxic to the cell. Pth cleaves the ester bond between tRNA and the peptide, thus preventing this toxicity and also releasing tRNA for further use. The enzyme from Mycobacterium tuberculosis (MtPth), is a 191-residue monomeric protein. We deter-

Keywords. Molecular crowding; molecular plasticity; molecular shape; peptidyl-tRNA hydrolase; protein function Supplementary materials pertaining to this article are available on the Journal of Biosciences Website at http://www.ias.ac.in/jbiosci/dec2012/supp/ Selvaraj.pdf 
mined, to start with, the structures of three crystal forms of this protein (Selvaraj et al. 2006; 2007) as part of a larger programme on the structural biology of mycobacterial proteins (Vijayan 2005; Krishna et al. 2007; Kaushal et al. 2008; Roy et al. 2008; Prabu et al. 2009; Chetnani et al. 2010). The X-ray structure of the homologue from E.coli ( $E c$ Pth) was then already available (Schmitt et al. 1997). The C-terminal stretch of MtPth was disordered in the crystal structures. On the other hand, in the crystal structure of $E c$ Pth, the C-terminus of the molecule was bound to the peptide binding site of a neighbouring molecule. Thus, $M t$ Pth provided the structure of the apoenzyme while $E c \mathrm{Pth}$ presented a picture of the structure of the peptide bound enzyme.

The Pth molecule, with an $\alpha / \beta$ fold involving a twisted $\beta$ sheet flanked by helices, has two binding regions separated by a gate (figure 1). One of them, the peptide binding region, involves a lid containing a helix and a loop (Gly136 to Val150 in $M t$ Pth). This region is separated from the tRNA binding region by a gate made up of Asp98 and Gly113. In the MtPth structure without any ligand, the lid has an open conformation and the gate is closed. In the crystal structure of $E c \mathrm{Pth}$, where a peptide stretch is bound to the peptide binding region, the lid is closed on the bound peptide. Concurrently, the gate opens such that the peptide and the tRNA binding regions are now contiguous permitting the binding of peptidyl-tRNA to the molecule prior to hydrolysis. Thus, the $M t$ Pth and $E c$ Pth structures appeared to provide a plausible picture of functionally important plasticity in terms of the correlated motion of the lid and the gate (Selvaraj et al. 2007). This picture derived further support from the crystal structures of Mycobacterium smegmatis Pth (MsPth, Pdb code: 3p2j) and Francisella tularensis Pth (FtPth, Pdb code: 3 nea), which became available subsequently (Clarke et al. 2011). The molecule is unliganded in the MsPth structures and it has an open lid and a closed gate. The crystal structure of FtPth is similar to that of $E c$ Pth with the C-terminal peptide bound to a neighbouring molecule. As in the EcPth structure, the FtPth molecule has a closed lid and an open gate.

In the meantime, the solution NMR structure of MtPth became available. The overall structure of the molecule in solution is understandably similar to that in the crystals, although there are differences in detail. The C-terminal stretch of the molecule is disordered to different extents in the crystal structures while it forms a helix in solution. More strikingly, unlike in the crystal structures, the gate is open in the solution NMR structure although the lid has an open conformation in the crystals and solution (Pulavarti et al. 2009). To further address this anomaly, more crystal forms of $M t \mathrm{Pth}$, grown under different conditions, were $\mathrm{X}$-ray analysed (Selvaraj et al. 2012). As in the case of the MtPth crystals analysed earlier, the molecule in them had an open lid and a closed gate. Thus, all the crystal structures of Pth from different sources analysed so far give indication for a functionally important inverse correlation between the locations of the lid and the gate. Such a correlation does not exist in the solution NMR structure (Selvaraj et al. 2012). As a further effort, molecular dynamics (MD) simulations were undertaken on $M t$ Pth and EcPth. The results of these simulations, reported here, along with the available X-ray and NMR results appear to indicate a relation among crowding, macromolecular volume and plasticity. Subsequently, proteins for which X-ray as well as NMR results were available were carefully examined. Earlier studies involving such results have led to concerns about packing in NMR structures (Ratnaparkhi et al. 1998; Fu et al. 2011; Schwieters and Clore 2008). The fairly comprehensive examination
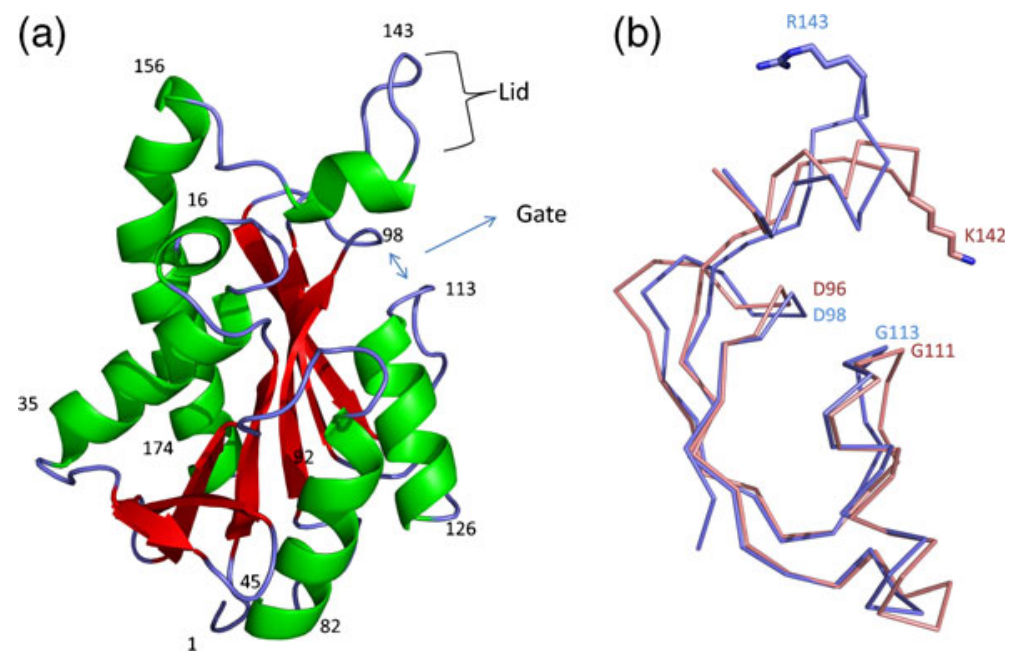

Figure 1. (a) Overall architecture of $M t \mathrm{Pth}$, with lid and gate highlighted. (b) Close up view of the active site of $E c \mathrm{Pth}$ (pink) and $M t \mathrm{Pth}$ (blue). 
presented here focuses specifically on macromolecular volume and the space available around the protein molecule. This examination appears to lend additional support to the hypothesis on the relation mentioned above.

\section{Methods}

Molecular dynamics (MD) simulations were performed using the package GROMACSv3.3.1 running on parallel processor with OPLS-AA/L force field (Jorgensen et al. 1996; Spoel et al. 2005). Initially, the crystallographic water molecules in the structures were removed from the protein model. The conformation of lids in $M t$ Pth and EcPth was interchanged using COOT. A cubic box around the protein molecule was generated using the editconf module of GROMACS with the criterion that the minimum distance between the solute and the edge of the box was at least $7.5 \AA$. Following this, the protein models were solvated with TIP4P water model using program genbox available in the GROMACS suite. Sodium or chloride ions were added to neutralize the overall charge of the system whenever necessary. Energy minimizations were carried out using the conjugate gradient and steepest descent methods with a frequency of latter at 1 in 1000. A maximum force of $1 \mathrm{kJmol}^{-1} \mathrm{~nm}^{-1}$ was chosen as convergence criterion for minimization. Energy minimizations were followed by solvent equilibration by position restrained dynamics of $10 \mathrm{ps}$ where positions of protein atoms were restrained and solvent was allowed to move. Simulations utilized the NPT ensembles with Parrinello-Rahman isotropic pressure coupling $\left(\tau_{\mathrm{p}}=0.5\right)$ to 1 bar and Nose-Hoover temperature coupling $\left(\tau_{\mathrm{t}}=0.1 \mathrm{ps}\right)$ to $300 \mathrm{~K}$. Long-range electrostatic interactions were computed using the Particle Mesh Ewald (PME) method with a cut-off of $12 \AA$ (Darden et al. 1993). A cut-off of $15 \AA$ was used to compute long-range van der Waals interactions. Simulations were performed with full periodic boundary conditions ( $\mathrm{PBC}$ ). Bonds were constrained with the LINCS algorithm (Berk Hess et al. 1997). Simulations were carried out with a dielectric constant of unity.

Structure superpositions were performed using ALIGN (Cohen 1997). Principal axes of the ellipsoidal representation of the protein molecules were calculated using CHIMERA (Pettersen et al. 2004). The ellipsoidal volume of the molecules was calculated using the principal axes.

\section{Results and discussion}

\subsection{MD simulations}

To obtain an additional independent insight into the structure and plasticity of $M t \mathrm{Pth}$, MD simulations were carried out on the molecule. A molecule obtained from form I was used as the starting model (Selvaraj et al. 2007). The C-terminal stretch involving residues from 180 to 191 was not defined in this form. The structure of the stretch in the crystal structure of $E c$ Pth was used to complete the model. Simulations were also performed with $E c$ Pth as the starting model. In order to explore the effect of starting models on the outcome of simulations, similar calculations were carried out with $M t \mathrm{Pth}$ having a closed lid conformation as in $E c$ Pth and $E c$ Pth with an open lid conformation as in MtPth, as starting models. Each simulation was run for $60 \mathrm{~ns}$. The trajectory of r.m.s. deviations in them are given in the supplementary figure 1.

The root mean square fluctuations of $\mathrm{C} \alpha$ positions along the polypeptide chain obtained from MD simulations are given in figure 2 . The pattern of the fluctuation is nearly the same in the four simulations. The nature of the flexibility of the molecule indicated by the simulations is nearly the same as that shown by the X-ray and the NMR results (Selvaraj et al. 2012). The C-terminal stretch is the most flexible region of the molecule. This flexibility has been suggested to be of biological significance (Selvaraj et al. 2012). The lid region (residues 136 to 150), which has an open conformation in the crystal structures in the absence of a bound ligand and closed conformation when bound to a peptide, also exhibits substantial flexibility. One of the gate residues, Gly113, which again moves in crystal structures, is in a flexible region. Another flexible stretch is the 50-60 loop. The biological significance of the flexibility of this loop is unclear. In the three-dimensional structure, this loop is flanked by the highly flexible C-terminal stretch and the moderately flexible $\mathrm{N}$-terminal region.

\subsection{Movement of the lid and the gate}

The movement of the lid is best described in terms of the distance between the $\mathrm{C} \alpha$ of Val92, which is an invariant residue in the rigid core of the molecule, and the $\mathrm{C} \alpha$ of Arg143 (Lys142/143 in EcPth and FtPth) at the tip of the lid (Selvaraj et al. 2012). The distance between the $\mathrm{C} \alpha$ atoms of Asp98 and Gly113 can be obviously used to describe the movement of the gate. The lid is open and the $92 \mathrm{C} \alpha-143 \mathrm{C} \alpha$ distance in the relevant mycobacterial structures varies between $28.0 \AA$ and $29.4 \AA$. The gate in these structures is closed with a $98 \mathrm{C} \alpha-113 \mathrm{C} \alpha$ distance varying between $6.1 \AA$ and $7.4 \AA$. The lid is closed in EcPth and FtPth with a lid distance of $23.6 \AA$ and $23.4 \AA$, respectively. The distance between $98 \mathrm{C} \alpha$ and $113 \mathrm{C} \alpha$ in the open gate in the two structures is $9.5 \AA$ and $10.0 \AA$, respectively. The distances are thus inversely correlated in the crystal structures (figure 3a). The lid distances of the 40 models obtained from NMR range between $25.3 \AA$ to $31.7 \AA$ with an average value of $28.5 \AA$, a value appropriate for an open conformation (Selvaraj et al. 2012). The gate is always open. The distance between $98 \mathrm{C} \alpha$ and $113 \mathrm{C} \alpha$ varies between $8.6 \AA$ and $17.7 \AA$ with an average value of $12.6 \AA$. The correlation between the distances is absent or is very weak at best (figure $3 b$ ). 


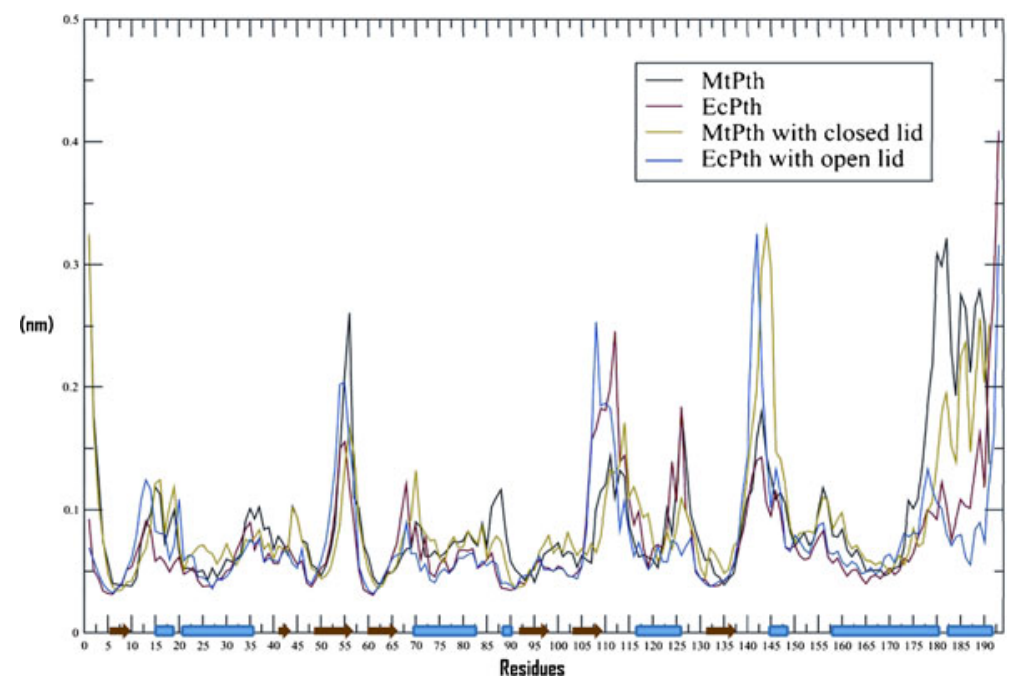

Figure 2. Root mean square fluctuations of simulated structures with the secondary structural elements of the molecule indicated at the bottom of the figure.

The simulations indicate two possible gate distances, one around $10.5 \AA$ and the other around $14 \AA$ (figure $4 \mathrm{a}$ ), the latter being the prominent one except in $E c$ Pth. Even in $E c$ Pth, the distance eventually stabilizes at around $14 \AA$ (figure $4 \mathrm{c}$ ). On the contrary, the lid distance appears to critically depend on the starting model. In three out of the four cases, the distance hovers around the value in the starting model (figure $4 \mathrm{~b}$ ). The distance exhibits a wide scatter in the simulation of MtPth with a closed lid. Even in this case, the distance eventually stabilizes at values close to that in the starting model (figure 4d). Irrespective of the population distribution and the time evolution of the gate and the lid distances, there is no appreciable correlation between them in any of the four simulations (figure 5).

\subsection{Volume of the molecule and the available space around it}

The apparent discrepancy between the X-ray and the NMR results on lid and gate distances in MtPth was a cause for concern. MD simulations, like X-ray and NMR results, indicated an open conformation for the lid, but yielded gate distances even larger than those obtained through NMR. The crystal structures of Pths showed an inverse correlation between lid distance and gate distance, while NMR studies and MD simulations of MtPth indicated hardly any correlation between the two. There was no obvious explanation for this intriguing situation. A careful visual examination appeared to suggest that the Pth molecule(s) derived from NMR studies was slightly larger than that seen in crystal structures. To further explore this possibility, ellipsoidal volumes of the molecule in the relevant crystal structures, the NMR structures, and in the course of MD simulations were calculated, along with the principal axes of the ellipsoids (table 1).

It is clear from table 1 that the ellipsoidal volume of the molecule obtained from NMR studies is higher than that obtained from crystal structures. The MD simulations yield an intermediate value. The change in volume appears to be related to the volume of the solution around the molecule (table 1). The unit cell of the crystal containing four molecules has a volume of $1.66 \times 10^{5} \AA^{3}$ with the solvent content (a)

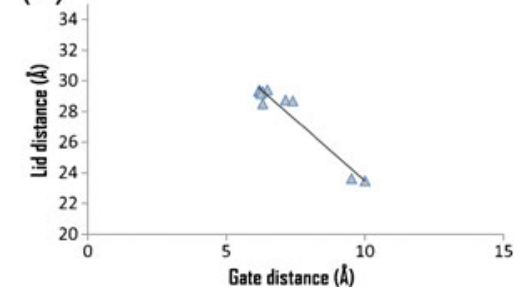

(b)

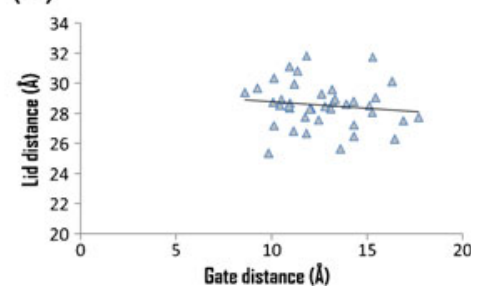

Figure 3. Correlation between gate and lid distances in (a) available crystal structures and (b) NMR structures. Reproduced with permission from the International Union of Crystallography from Selvaraj et al. (2012) (http://journals.iucr.org/). 
(a)

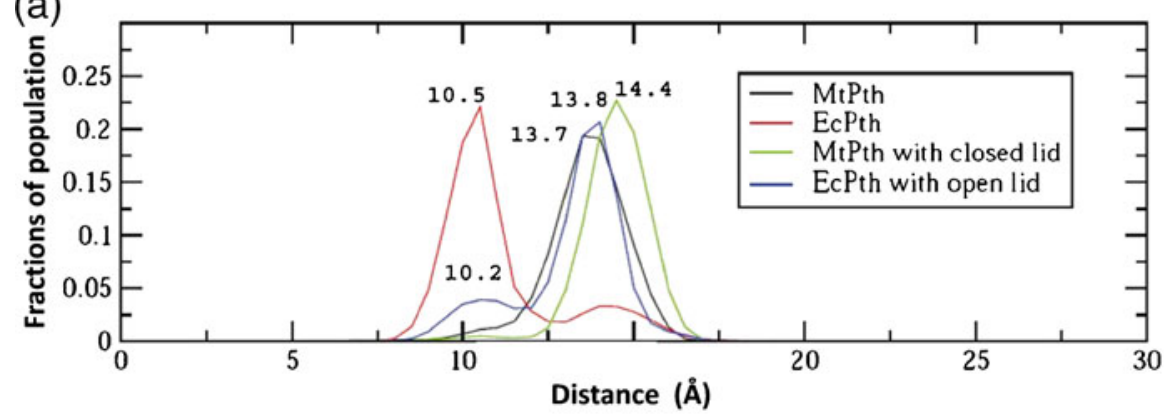

(b)

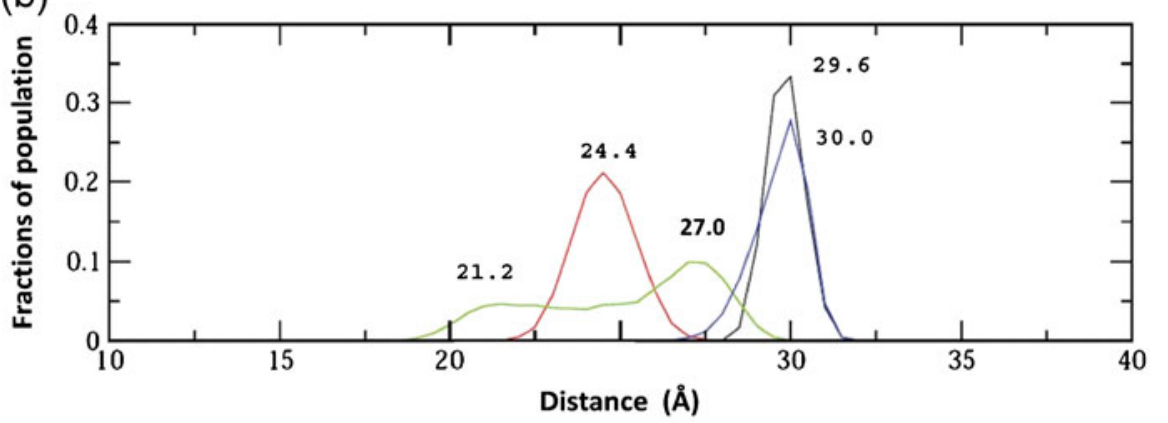

(c)

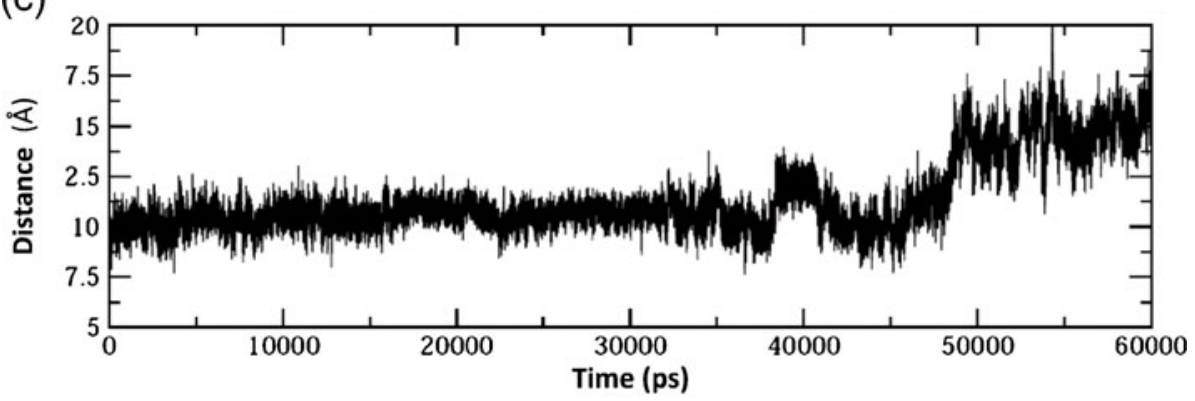

(d)

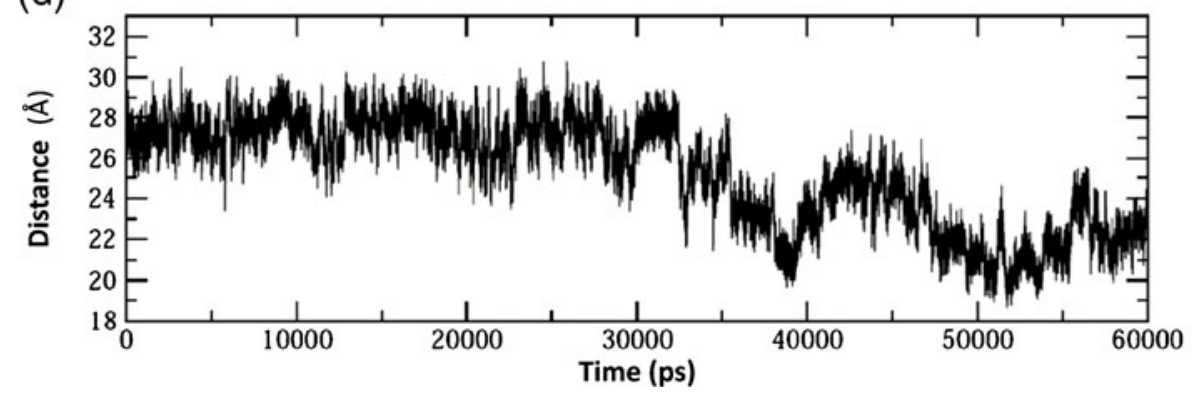

Figure 4. Population distributions of (a) the gate and (b) the lid distances in MD simulations. The time evolution of (c) the gate distance in the simulation of $E c$ Pth and (d) the lid distance in the MtPth with a closed lid.

occupying about $40 \%$ of the volume as estimated by the Matthews method (Matthews 1968). Thus, the volume of the solvent around the molecule in the crystal is less than the volume of the molecule. The volume of water surrounding the one molecule in the box in which MD simulations were carried out $\left(3 \times 10^{5} \AA^{3}\right)$ is more than an order of magnitude higher. At a concentration of $0.8 \mathrm{mM}$ used in the experiment, the volume of the solution per molecule in the medium in which the NMR experiments were performed $\left(2 \times 10^{6} \AA^{3}\right)$ works out to be still higher by close to one more order of magnitude (Bal et al. 2006). Thus there appears to be a correlation, though not linear, between the ellipsoidal volume of the molecule and that of the surrounding medium. 

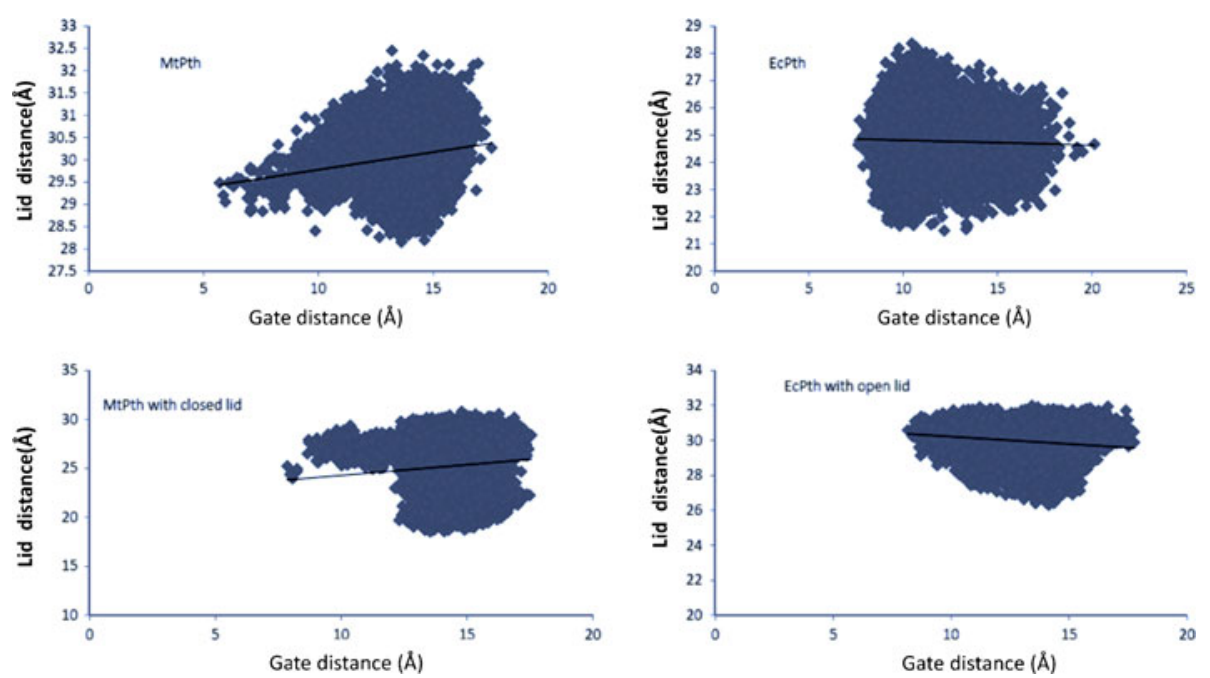

Figure 5. Correlation between gate and lid distances in simulations.

Protein structures can often be described as made up of a core region involving the basic fold elaborated through the addition of loops and other secondary structural elements. It was important to inquire if the correlation mentioned above holds even when the core region alone is considered. According to the SCOP classification (Murzin et al. 1995), MtPth has a phosphorylase/hydrolase-like fold involving a twisted $\beta$-sheet (residues 6-9, $41-42,49-55,59-63,90-96,105-108$ and $131-136$ ) flanked by two helices on either side (22-35 and 156-159, and 69-83 and 116-125) (figure 1a). Among these, 9 Nterminal residues of the long 156-179 helix juts out of the bulk of the molecule. The basic fold minus these nine residues can be considered as constituting the core of the molecule made up of close to 80 residues. The ellipsoidal volumes of this core in the X-ray, MD and NMR structures were also compared (table 1). The correlation exhibited by the volumes of the whole molecule is seen in the volumes of the core region as well, adding credence to the hypothesis regarding the relation between the ellipsoidal volume of the molecule and the volume of the surrounding medium. In the three situations considered, only in the crystals molecules and the surrounding medium have comparable volumes and each molecule is in contact with surrounding molecules. Does such crowding in general lead to a reduction in molecular volume as in the case of Pth? Is such crowding required for the correlated movement necessary for enzyme action, to manifest itself?

\subsection{A comparative survey of X-ray and NMR structures}

In order to address the first question posed above, the structures of proteins determined by X-ray as well as NMR were examined. 1271 such structures are available in the PDB (Berman et al. 2002) as on May 31st, 2012. This data base covers a wide range of folds involving $\alpha, \beta$, and $\alpha / \beta$ proteins and also different families with a variety of functions. Among the X-ray and NMR structures in the database, 533 are not directly comparable as the structure determinations involve different segments of the protein molecule. Ellipsoidal volumes of the X-ray and NMR structures were compared in all

Table 1. Ellipsoidal volume of the protein molecule along with the principal axes and the volume of the solvent around the molecule. The Cterminal stretch 180-191 has been removed from all the calculations as it is partly or wholly disordered in crystal structures (See text for details)

\begin{tabular}{|c|c|c|c|c|c|c|c|}
\hline \multirow[b]{2}{*}{ S. No. } & \multirow[b]{2}{*}{ Structure } & \multicolumn{3}{|c|}{ Principal axes of ellipsoid ( $\AA$ ) } & \multirow{2}{*}{$\begin{array}{l}\text { Ellipsoidal volume } \\
\text { of the molecule }\left(\AA^{3}\right)\end{array}$} & \multirow{2}{*}{$\begin{array}{l}\text { Volume of solvent } \\
\text { around the molecule }\left(\AA^{3}\right)\end{array}$} & \multirow{2}{*}{$\begin{array}{l}\text { Ellipsoidal volume } \\
\text { of the core of the } \\
\text { molecule }\left(\AA^{3}\right)\end{array}$} \\
\hline & & $\mathrm{a}$ & $\mathrm{b}$ & $\mathrm{c}$ & & & \\
\hline 1 & Form I $M t$ Pth & 24.472 & 17.629 & 15.772 & 28,513 & 18,893 & 16,719 \\
\hline 2. & $\begin{array}{l}\text { MtPth MD structure } \\
\text { (at } 5000 \mathrm{ps} \text { ) }\end{array}$ & 25.356 & 17.912 & 15.959 & 30,373 & $3,00,675$ & 17,432 \\
\hline 3. & $\begin{array}{l}\text { MtPth NMR structure } \\
\text { (Model 1) }\end{array}$ & 24.974 & 19.267 & 16.267 & 32,799 & $20,42,578$ & 19,364 \\
\hline
\end{tabular}


Table 2. Ellipsodial volume of the molecule and volume of solvent around the molecule in directly comparable X-ray and NMR structures (See text for details)

\begin{tabular}{|c|c|c|c|c|c|c|c|c|}
\hline \multirow[b]{2}{*}{ S.No. } & \multirow[b]{2}{*}{ Protein } & \multicolumn{2}{|c|}{ PDB code } & \multicolumn{2}{|c|}{$\begin{array}{c}\text { Ellipsoidal } \\
\text { volume of the } \\
\text { molecule }\left(\AA^{3}\right)\end{array}$} & \multirow{2}{*}{$\begin{array}{c}\text { Unit cell }^{3} \\
\text { volume }(\AA)\end{array}$} & \multicolumn{2}{|c|}{$\begin{array}{l}\text { Volume of solvent } \\
\text { around the } \\
\text { molecule }\left(\AA^{3}\right)\end{array}$} \\
\hline & & X-ray & NMR & $\mathrm{X}$-ray & NMR & & X-ray & NMR \\
\hline 1 & NEDD4 binding protein from Homo sapiens & 3FAU & 2D9I & 12687 & 14210 & 139205 & 24053 & 1646092 \\
\hline 2 & Basic fibroblast growth factor from Homo sapiens & 1BAS & 1BLA & 19291 & 22980 & 33012 & 17481 & 1637322 \\
\hline 3 & Replication protein A from Homo sapiens & 2B29 & $1 \mathrm{EWI}$ & 20039 & 23916 & 183091 & 16091 & 1636386 \\
\hline 4 & Interleukin enhancer-binding factor 3 from Homo sapiens & 3P1X & $2 \mathrm{~L} 33$ & 11256 & 14452 & 154204 & 22293 & 2357408 \\
\hline 5 & APOA-1 from Homo sapiens & 1AV1 & $1 \mathrm{GW} 3$ & 11868 & 18084 & 2177494 & 143830 & 313976 \\
\hline 6 & Tyrosine protein phosphatase type 4 from Homo sapiens & $2 \mathrm{VPH}$ & $2 \mathrm{CS} 5$ & 16465 & 18751 & 185529 & 21409 & 903639 \\
\hline 7 & $\begin{array}{l}\text { Spliceosomal U5 snRNP specific } 15 \mathrm{kDa} \text { protein from } \\
\text { Homo sapiens }\end{array}$ & 1QGV & $1 \mathrm{PQN}$ & 21219 & 24556 & 144565 & 19006 & 1635746 \\
\hline 8 & Peptidyl-tRNA hydrolase 2 from Archaeoglobus fulgidus & 3ERJ & $1 \mathrm{RZW}$ & 20264 & 23576 & 216857 & 23613 & 1636726 \\
\hline 9 & CzrA protein from Staphylococcus aureus & 1R1U & $2 \mathrm{KJB}$ & 22309 & 31595 & 333807 & 12593 & 1628707 \\
\hline 10 & Alpha-amylase from Elusine coracana & $1 \mathrm{~B} 1 \mathrm{U}$ & $1 \mathrm{BIP}$ & 19224 & 25386 & 104153 & 55172 & 804765 \\
\hline 11 & Neuronal bungarotoxin from Bungarus multicinctus & $1 \mathrm{KBA}$ & $2 \mathrm{NBT}$ & 10958 & 14488 & 220588 & 21089 & 193049 \\
\hline 12 & Interleukin-2 from Homo sapiens & $3 \mathrm{INK}$ & 1IRL & 21991 & 25314 & 71045 & 36025 & 528120 \\
\hline 13 & Homeobox protein engrailed from Homo sapiens & $1 \mathrm{ENH}$ & 1ZTR & 10139 & 12989 & 204124 & 9836 & 2754181 \\
\hline 14 & Arc represson from phage $\mathrm{P} 22$ & 1BAZ & 1ARQ & 15371 & 17034 & 228611 & 32607 & 621543 \\
\hline 15 & Regulatory protein E2 from Bovine papillomavirus type 1 & $1 \mathrm{JJH}$ & 1DBD & 13679 & 16093 & 1101126 & 62755 & 774527 \\
\hline 16 & Interleukin-4 from Homo sapiens & 1HIJ & $1 \mathrm{BBN}$ & 23513 & 25870 & 393583 & 32175 & 804281 \\
\hline 17 & Excinuclease ABC subunit from Escherchia coli & 1QOJ & $1 \mathrm{E} 52$ & 6183 & 7749 & 331332 & 46182 & 1652553 \\
\hline 18 & Spectrin alpha chain from Gallus gallus & 1BK2 & 2LJ3 & 9201 & 11706 & 70008 & 10602 & 1648596 \\
\hline 19 & Prolifin1A from Acanthamoeba $s p$. & $1 \mathrm{ACF}$ & $2 \mathrm{PRF}$ & 18751 & 21662 & 108528 & 27132 & 1255493 \\
\hline 20 & Enzyme -I from Escherchia coli & $1 Z Y M$ & $1 \mathrm{EZA}$ & 50615 & 56113 & 611899 & 86220 & 1050755 \\
\hline 21 & Apoliphophorin-III from Locusta migratoria & 1AEP & 1LS4 & 24989 & 30428 & 613983 & 32548 & 1629874 \\
\hline 22 & CAMP- dependent kinase type II from Mus musculus & $2 \mathrm{IZY}$ & $1 \mathrm{R} 2 \mathrm{~A}$ & 9671 & 12363 & 1370428 & 57982 & 817788 \\
\hline 23 & Cardiotoxin V from Geobacillus stearothermophilus & $1 \mathrm{KXI}$ & $1 \mathrm{CVO}$ & 9754 & 10868 & 277108 & 19235 & 404207 \\
\hline 24 & Beta-elicitin cryptogein from Phytoptora cryptogea & $1 \mathrm{BEO}$ & $1 \mathrm{BEG}$ & 15068 & 17656 & 291813 & 25088 & 535778 \\
\hline 25 & Fimbrial protein from Pseudomonas aeruginosa & 1QVE & 1HPW & 22976 & 28044 & 49568 & 20018 & 3292560 \\
\hline 26 & P85-alpha from Bos taurus & 1QAD & 1BFI & 18301 & 20491 & 114300 & 15336 & 1639811 \\
\hline 27 & YfjZ from E.coli & 2EA9 & $2 \mathrm{JN} 7$ & 16734 & 24794 & 82993 & 14969 & 1484571 \\
\hline 28 & $\begin{array}{c}\text { Mesencephalic astrocyte-derived neurotrophic factor } \\
\text { from Homo sapiens }\end{array}$ & $2 \mathrm{~W} 51$ & $2 \mathrm{KVD}$ & 34240 & 38408 & 282671 & 32141 & 1621894 \\
\hline 29 & HNRNP K from Homo sapiens & $1 \mathrm{ZZK}$ & $1 \mathrm{KHM}$ & 14974 & 17213 & 66226 & 6591 & 1643089 \\
\hline 30 & $\begin{array}{l}\text { Peptidyl-tRNA hydrolase from Mycobacterium } \\
\text { tuberculosis }\end{array}$ & 2Z2I & $2 \mathrm{JRC}$ & 28513 & 32799 & 166064 & 18893 & 2042578 \\
\hline 31 & ORF c02003 protein from Sulfolobus solfataricus & 2Q00 & 2JPU & 19720 & 24689 & 1367409 & 53363 & 1484676 \\
\hline 32 & Q251Q8_DESH from Desulfitobacterium hafniense & 3IPF & $2 \mathrm{KS} 0$ & 10226 & 12477 & 557575 & 28911 & 1647825 \\
\hline 33 & Plectasin from Sulfolobus solfataricus & $3 \mathrm{E} 7 \mathrm{R}$ & $1 \mathrm{ZFU}$ & 4959 & 5679 & 12707 & 9066 & 1654623 \\
\hline 34 & Sso10a from Sulfolobus solfataricus & 1R7J & $1 \mathrm{XSX}$ & 20908 & 23793 & 126886 & 20134 & 667999 \\
\hline 35 & Riboflavin kinase from Methanocaldococcus jannaschii & 2VBS & $2 \mathrm{P} 3 \mathrm{M}$ & 24446 & 31833 & 646412 & 62956 & 2522477 \\
\hline 36 & Replication protein A from Methanococcus maripaludis & $3 \mathrm{E} 0 \mathrm{E}$ & $2 \mathrm{~K} 5 \mathrm{~V}$ & 14717 & 16210 & 77972 & 9119 & 1390825 \\
\hline 37 & Lipoprotein from Neisseria meningitidis & $3 \mathrm{KVD}$ & $2 \mathrm{KC} 0$ & 46282 & 51917 & 426388 & 40825 & 4098838 \\
\hline 38 & PSPTO_3016 protein from Pseudomonas syringae & $3 \mathrm{H} 9 \mathrm{X}$ & $2 \mathrm{KFP}$ & 21548 & 23895 & 145762 & 79246 & 1485470 \\
\hline 39 & UPF0352 protein from Shewanella oneidensis & 2QTI & 2JUW & 22185 & 24819 & 159942 & 13283 & 1635483 \\
\hline 40 & Putative Diflavin flavoprotein A3 from Nostac sp. & 3FNI & $2 \mathrm{KLB}$ & 25254 & 29223 & 139988 & 15224 & 1377812 \\
\hline 41 & Putative chaperone Q8ZP25 from Salmonealla & 2ES7 & 2GZP & 19414 & 23726 & 267354 & 75804 & Not available \\
\hline
\end{tabular}


Table 2 (continued)

\begin{tabular}{|c|c|c|c|c|c|c|c|c|}
\hline \multirow[b]{2}{*}{ S.No. } & \multirow[b]{2}{*}{ Protein } & \multicolumn{2}{|c|}{ PDB code } & \multicolumn{2}{|c|}{$\begin{array}{c}\text { Ellipsoidal } \\
\text { volume of the } \\
\text { molecule }\left(\AA^{3}\right)\end{array}$} & \multirow{2}{*}{$\begin{array}{c}\text { Unit cell }^{3} \\
\text { volume }(\AA)\end{array}$} & \multicolumn{2}{|c|}{$\begin{array}{c}\text { Volume of solven } \\
\text { around the } \\
\text { molecule }\left(\AA^{3}\right)\end{array}$} \\
\hline & & X-ray & NMR & $\mathrm{X}$-ray & NMR & & X-ray & NMR \\
\hline 42 & Lin2157 protein from Listeria innocua & $3 \mathrm{IIE}$ & $2 \mathrm{KJK}$ & 13736 & 15332 & 125747 & 17901 & 1391703 \\
\hline 43 & $\begin{array}{c}\text { Conserved hypothetical protein PA2412 from } \\
\text { Pseudomonas aeroginosa }\end{array}$ & 2PST & $2 \mathrm{GPF}$ & 10190 & 13857 & 86594 & 14475 & 2753313 \\
\hline 44 & Conserved lipoprotein from Ureaplasma parvum & $3 \mathrm{JVC}$ & $2 \mathrm{KRT}$ & 19558 & 22802 & 833565 & 57268 & 1821978 \\
\hline 45 & Nudix hydrolase DR0079 from Deinococcus radiodurans & $2 \mathrm{O} 5 \mathrm{~F}$ & 1Q27 & 28837 & 33020 & 674034 & 40819 & 797131 \\
\hline 46 & DR_A0006 from Deinococcus radiodurans & $3 \mathrm{GGN}$ & $2 \mathrm{KCZ}$ & 19740 & 22137 & 134396 & 31713 & 1822643 \\
\hline 47 & Coactosin like protein from Homo sapiens & $1 \mathrm{~T} 2 \mathrm{~L}$ & 1TMW & 22533 & 26598 & 265180 & 32102 & 1080270 \\
\hline 48 & Acylphophatase 1 from Homo sapiens & $2 \mathrm{~W} 4 \mathrm{C}$ & $2 \mathrm{~K} 7 \mathrm{~K}$ & 15040 & 17906 & 84128 & 8247 & 2749264 \\
\hline
\end{tabular}

the remaining 738. The ellipsoidal volume of the X-ray and the corresponding NMR structures differed by $10 \%$ or more in 206 proteins. A $10 \%$ difference was taken as highly significant and these 206 structures were carefully examined. In 158 cases, the $\mathrm{X}$-ray and the NMR were not again directly comparable as there were differences in bound ligand, length of defined

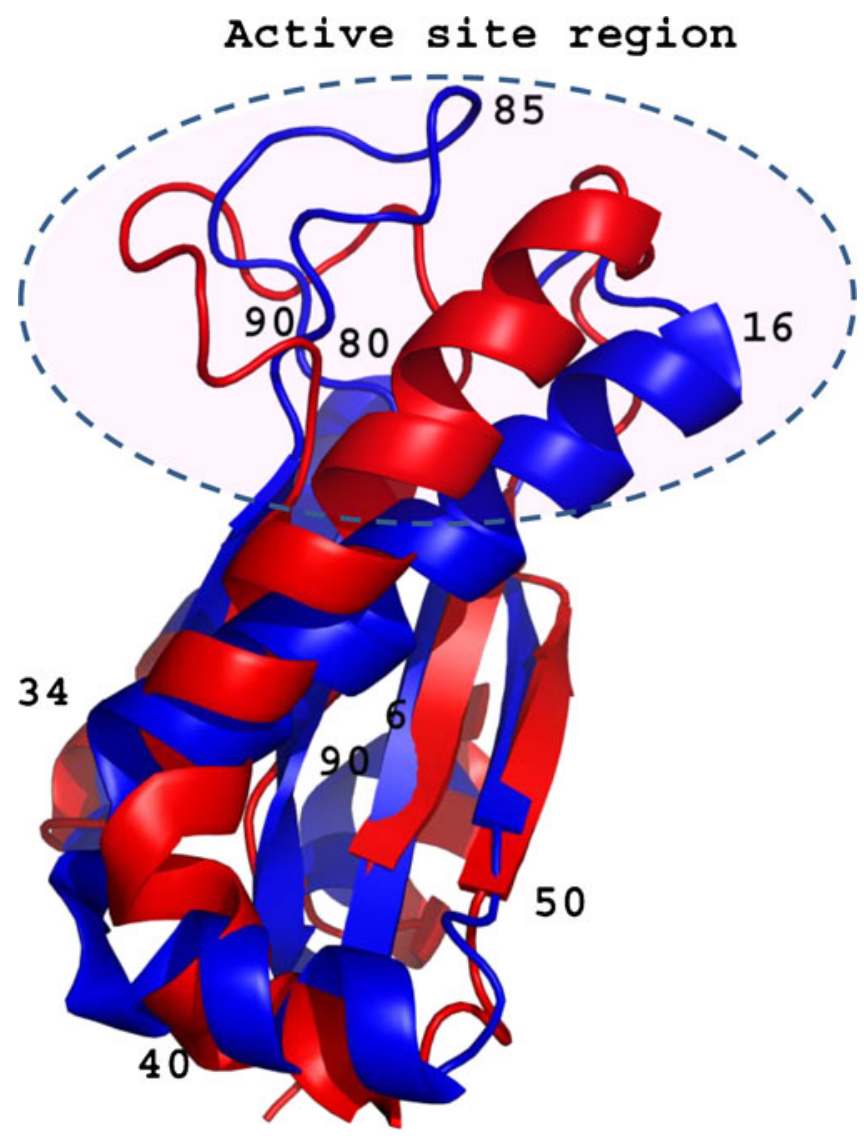

Figure 6. X-ray structure of AfPth2 (blue) superposed over the NMR structure of AfPth2 (red). polypeptide chain, domain swapping, etc. In the remaining 48 , which were comparable in almost every aspect, the NMR structure was larger than the X-ray structure in volume by $10 \%$ or more (table 2). Thus, it appeared that, in general, the ellipsoidal volumes of NMR structures are larger than those of the corresponding X-ray structures.

It was important to examine if the larger volume of NMR structures is caused by environmental effects. The composition of the crystallization medium and the NMR solvent vary so widely that it is unlikely to lead to a systematic effect. The situation is somewhat different in relation to temperature. Most X-ray structures have been determined at low temperature whereas most NMR structures have been elucidated at room temperature. There are, however, three structures [X-ray (NMR) structure Pdb codes: $2 \mathrm{w} 4 \mathrm{c}(2 \mathrm{k} 7 \mathrm{k}), 1 \mathrm{prq}$ (2prf) and 1a43 (2lf4)], which have been determined crystallographically and by NMR at the same or nearly the same temperature (Worthylake et al. 1999; Bennet et al. 2003; Lam et al. 2011). In all the three instances, the ellipsoidal volume of the NMR structure is more than $10 \%$ larger than that of the X-ray structures as in the case of structures determined at different temperatures using $\mathrm{X}$-ray and NMR. Furthermore, there are instances where the same protein crystals in the same space group grown under the same or similar conditions have been studied at different temperatures. For example, the ribosome recycling factor from $M$. tuberculosis has almost the same ellipsoidal volume in its crystal at $100 \mathrm{~K}(1 \mathrm{wqg})$ and at room temperature (1wqf) (Saikrishnan et al. 2005). In the case of M. smegmatis RecA crystals, the volume at low temperature (2zrn) is slightly higher than at room temperature (2zr7) (Prabu et al. 2008). It would thus appear that difference in temperature has only a minor effect on the volume. However, the solvent volume available for each molecule in the crystal is roughly the same as the molecular volume, whereas it is one or two orders of magnitude higher in solutions used for NMR experiments. Thus, the lower molecular volume found in crystal structures, in comparison with that obtained from 
NMR studies in solution, is most probably on account of macromolecular crowding.

Among the other proteins for which X-ray and NMR structures are available, there appears none to be directly comparable to eubacterial Pth with concerted motion of two regions of the molecule. The closest to eubacterial Pth among them is an archeal Pth. Archaeal and eukaryotic Pths, often referred to as Pth2, have similar structures which are very different from the structures of eubacterial Pth. The X-ray structures of dimeric Pth2 from Archaeglobus flucidus (3erj), Sulfolobus solfataricus (1xty), Pyrococcus horikoshii (1wn2) and humans (1q7s) are available (Pereda et al. 2003; Fromant et al. 2005; Shimuzu et al. 2008). These proteins which share $40 \%$ to $52 \%$ sequence identity have very similar structures. The NMR structure of AfPth2 is also available (Powers et al. 2005). A superposition of X-ray and NMR structures of AfPth2 is shown in figure 6. Although the rest of the structure superposes well, the active site region involving a loop and one end of the longest helix in the structure exhibit substantial differences. The loop has an open conformation in the NMR structure while it closes on the main body of the molecule in the X-ray structure. The end of the helix that forms part of the active site moves towards the rest of the molecule in the X-ray structure from its position in the NMR structure. Thus, as in the case of $M t \mathrm{Pth}, A f \mathrm{Pth} 2$ has a more voluminous open structure in solution than in crystals (table 2). A similar difference between NMR and X-ray structures involving a functionally important helix or loop has been reported in human hDim1 (Pdb codes 1qgv and 1pqn) (Reuter et al. 1999; Zhang et al. 2003) and a CTP-dependent archaeal riboflavin kinase as well (Pdb codes 2vbs and 2p3m) (Ammelburg et al. 2007).

\section{Conclusion}

The results presented here indicate that the molecular volume of proteins is dependent on the volume of the surrounding medium. The volume tends to be larger in solutions used in NMR experiments than in crystals where crowding exists. The recent observation that phosphoglycerate kinase has a more compact structure in cellular compartment than in aqueous solution, is of interest in this context (Dhar et al. 2011). It has been shown that the protein molecule could change its shape in a crowded environment (Homouz et al. 2008). The comparative study of NMR and X-ray structures indicate that the change in shape could specifically involve regions of the molecule important in activity, as indeed has been suggested by an earlier MD and theoretical investigation as well (Dong et al. 2010). The study also suggests that the functionally important plasticity of protein molecules could be modulated by crowding. This is in consonance with the observations of the effect of crowding on enzyme kinetics (Norris and Malys 2011). The effect of crowding on the structure, folding and action of proteins is complex and is characterized by subtlety and it needs to be pursued more extensively. However, the work reported here, in conjunction with results available in the literature, suggest that crowding leads to compaction of protein structure and influences the functionally important plasticity of the molecule.

\section{Acknowledgements}

Financial support from the Department of Biotechnology (DBT is acknowledged. Part of the computations was performed at the Interactive Graphics Facility supported by the DBT. MV is a DAE Homi Bhabha Professor.

\section{References}

Ai X, Zhou Z, Bai Y and Choy WY $2006{ }^{15} \mathrm{~N}$ NMR spin relaxation dispersion study of the molecular crowding effects on protein folding under native conditions. J. Am. Chem. Soc. 1263916 3917

Ammelburg M, Hartmann MD, Djuranovic S, Alva V, Koretke KK, et al. 2007 A CTP-dependent archaeal riboflavin kinase forms a bridge in the evolution of cradle-loop barrels. Structure 15 1557-1590

Bahadur RP, Chakrabarthi P, Rodier F and Janin J 2003 A dissection of specific and non-specific protein-protein interfaces. J. Mol. Biol. 336 943-955

Bal NC, Agrawal H, Meher AK, Pulavarti SV, Jain A, Kelley G, et al. 2006 NMR assignment of peptidyl-tRNA hydrolase from Mycobacterium tuberculosis H37Rv. J. Biomol. NMR 36 Suppl 1:53

Bennet EM, Li C, Allan PW and Ealick SE 2003 Structural basis for substrate specificity of Escherichia coli purine nucleoside phosphorylase. J. Biol. Chem. 278 47110-47118

Berk Hess HB, Berendsen HJC and Fraaije JGEM 1997 LINCS: A linear constraint solver for molecular simulations. J. Comput. Chem. 18 1463-1472

Berman HM, Battistuz T, Bhat TN, Bluhm WF, Bourne PE, Burkhardt K, et al. 2002 The Protein Data Bank. Acta Crystallog. Sect. D 58 899-90

Chetnani B, Kumar P, Surolia A and Vijayan M 2010 M. tuberculosis pantothenate kinase: Dual substrate specificity and unusual changes in ligand locations. J. Mol. Biol. 400 171-185

Clarke TE, Romanov V, Lam R, Gothe SA, Peddi SR, Razumova EB, et al. 2011 Structure of Francisella tularensis peptidyltRNA hydrolase. Acta Crystallog. Sect. F 67 913-915

Cohen GE 1997 ALIGN: a program to superimpose protein coordinates, accounting for insertions and deletions. J. Appl. Cryst. 30 1160-1161

Darden T, York D and Pedersen L 1993 Particle mesh Ewald: a $\mathrm{N} \cdot \log (\mathrm{N})$ method for Ewald sums in large systems. J. Chem. Phy. 98 10089-10092 
Dhar A, Girdhar K, Singh D, Gelman H, Ebbinghaus S and Gruebele M 2011 Protein stability and folding kinetics in the nucleus and endoplasmic reticulum of eukaryotic cells. Biophys. J. 101 421-430

Dong H, Qin S and Zhou HX 2010 Effect of macromolecular crowding on protein conformational changes. PLoS Comput. Biol. 6 e 1000833

Ellis RJ and Minton AP 2003 Join the crowd. Nature 425 27-28

Fromant M, Schmitt E, Mechulam Y, Lazennec C, Plateau P and Blanquet S 2005 Crystal structure at $1.8 \AA$ resolution and identification of active site residues of Sulfolobus solfataricus peptidyl-tRNA hydrolase. Biochemistry 44 4294-4301

Fu R, Wang X, Li C, Miranda AS, Pielak GJ and Tian F 2011 In situ structural characterization of a recombinant protein in native Escherichia coli membrane with solid state magic anlg spinning NMR. J. Am. Chem. Soc. 133 12370-12373

Gershenson A and Gierasch LM 2011 Protein folding in the cell: challenges and progress. Curr. Opin. Struct. Biol. 21 32-41

Homouz D, Perham M, Samiotakis A, Cheung MS and Pernilla WS 2008 Crowded, cell-like environment induces shape changes in aspherical protein. Proc. Natl. Acad. Sci. USA 105 11754-11759

Homouz D, Sanabria H, Waxham MN and Cheung MS 2009 Modulation of calmodulin plasticity by the effect of macromolecular crowding. J. Mol. Biol. 392 933-943

Jorgensen WL, Maxwell DS and Tirado-Rives J 1996 Development and testing of the OPLS all-atom force field on conformation energetic and properties of organic liquids. J. Am. Chem. Soc. 118 11225-11236

Kaushal PS, Talawar RK, Krishna PD, Varshney U and Vijayan M 2008 Unique features of the structure and interactions of mycobacterial uracil-DNA glycosylase: Structure of a complex of the Mycobacterium tuberculosis enzyme in comparison with those from other sources. Acta Crystallogr. Sect. D: Biol. Crystallogr. 64 551-560

Krishna R, Prabu JR, Manjunath GP, Datta S, Chandra NR, Muniyappa K and Vijayan M 2007 Snapshots of RecA protein involving movement of the $\mathrm{C}$-domain and different conformations of the DNA-binding loops: crystallographic and comparative analysis of 11 structures of Mycobacterium smegmatis RecA. J. Mol. Biol. 367 1130-1144

Lam SY, Yeung RC, Yu TH, Sze KH and Wong K 2011 A rigidifying salt-bridge favours the activity of thermophilic enzyme at high temperatures at the expense of low-temperature activity. PLoS Biol. 9 e1001027

Matthews BW 1968 Solvent content of protein crystals. J. Mol. Biol. 33 491-497

Murzin AG, Brenner SE, Hubbard T, Chothia C 1995 SCOP: a structural classification of proteins database for the investigation of sequences and structures. J. Mol. Biol. 247 536-540

Norris MGS and Malys N 2011 What is the true enzyme kinetics in the biological system? An investigation of macromolecular crowding effect upon enzyme kinetics of glucose-6-phosphate dehydrogenase. Biochem. Biophys. Res. Commun. 405 388-392

Pereda JM, Wass WF, Jan Y, Ruoslahti E, Schimmel P, and Pascual J 2003 Crystal structure of a human peptidyl-tRNA hydrolase reveals a new fold and suggests basis for a bifunctional activity. J. Biol. Chem. 279 8111-8115
Pernilla WS 2011 Protein folding inside the cell. Biophys. J. 101 265-266

Pettersen EF , Goddard TD, Huang CC, Couch GS, Greenblatt DM, Meng EC, et al. 2004 UCSF Chimera- a visualization system for exploratory research and analysis. J. Comput. Chem. 25 1605-1612

Powers R, Acton TB, Huang YJ, Liu J, Ma L, Rost B, et al. 2005 The solution structure of the Archaeglobus flugidis protein AF2095 Northeast structural genomics consortium target GR4. Protein Sci. 14 2849-2861

Prabu JR, Thamotharan S, Khanduja JS, Chandra NR, Muniyappa K and Vijayan M 2009 Crystallographic and modelling studies on Mycobacterium tuberculosis RuvA: Additional role of RuvBbinding domain and inter species variability. Biochim. Biophys. Acta 1794 1001-1009

Prabu JR, Manjunath GP, Chandra NR, Muniyappa K and Vijayan M 2008 Functionally important movements in RecA molecules and filaments: studies involving mutation and environmental changes. Acta Crystallogr. Sect. D 64 1146-1157

Pulavarti SV, Jain A, Pathak PP, Mahmood A and Arora A 2009 Solution structure and dynamics of peptidyl-tRNA hydrolase from Mycobacterium tuberculosis H37Rv. J. Mol. Biol. 378 165-177

Ratnaparkhi, GS, Ramachandran S, Udgaonkar, B and Varadarajan, R 1998 Discrepancies between the NMR and X-ray structures of uncomplexed Barstar: Analysis suggests that the packing densities of protein structures determined by NMR are unreliable. Biochemistry 37 6958-6966

Reuter K, Nottrot S, Fabrizio P, Luhrmann R and Fincer 1999 Identification, characterization and crystal structure analysis of the human spliceosomal U5 snRNP-specific $15 \mathrm{kD}$ protein. J. Mol. Biol. 294 515-525

Roque A, Ponte I, and Suau P 2007 Macromolecular crowding induces a molten globule state in the C-terminal domain of histone H1. Biophys. J. 93 2170-2177

Roy S, Saraswathi R, Chatterji D and Vijayan M 2008 Structural studies on the second Mycobacterium smegmatis Dps: Invariant and variable features of structure, assembly and function. J. Mol. Biol. 375 948-995

Saikrishnan K, Kalapala SK, Varshney U and Vijayan M 2005 Xray structural studies of Mycobacteriam tuberculosis RRF and a comparative study of RRFs of known structure. Molecular plasticity and biological implications. J. Mol. Biol. 345 29-38

Schmitt E, Mechulam Y, Fromant M, Plateau P and Blanquet S 1997 Crystal structure at $1.2 \AA$ resolution and active site mapping of Escherchia coli peptidyl-tRNA hydrolase. EMBO J. 16 4760-4769

Schwieters CD and Clore MG 2008 A pseudopotential for improving the packing of ellipsoidal protein structures determined from NMR data. J. Phys. Chem B 112 6070-6073

Selvaraj M, Ahmad R, Varshney U and Vijayan M 2012 Structures of new crystal forms of Mycobacterium tuberculosis peptidyltRNA hydrolase and functionally important plasticity of the molecule. Acta Crystallog. Sect. F 68 124-128

Selvaraj M, Roy S, Sangeetha R, Varshney U and Vijayan M 2007 Structural Plasticity and Enzyme Action: Crystal Structures of 
Mycobacterium tuberculosis Peptidyl-tRNA Hydrolase. J. Mol. Biol. 372 186-193

Selvaraj M, Singh NS, Roy S, Sangeetha R, Varshney U and Vijayan M 2006 Cloning, expression, purification, crystallization and preliminary X-ray analysis of peptidyl-tRNA hydrolase from Mycobaterium tuberculosis. Acta. Crystallog. Sect. F. 62 913-915

Shimuzu K, Kuroishi C, Sugahara M and Kunishima N 2008 Structure of peptidyl-tRNA hydrolase 2 from Pyrococcus horikoshii OT3: insight into the functional role of its dimeric state. Acta Crystallogr. Sect D 64 444-453

Spoel VD, Lindahl E, Hess B, Groenhof G, Mark AE and Berendsen HJ 2005 Gromacs: Fast, flexible, and free. J. Comput. Chem. 26 1701-1718
Vijayan M. Structural biology of mycobacterial proteins: The Bangalore effort 2005 Tuberculosis 85 357-366

Worthylake DK, Wang H, Yoo S, Sundquist WI and Hill CP 1999 Structures of HIV-1 capsid protein dimerizationdomain at $2.6 \AA$ resolution. Acta Crystallog. Sect. D 55 85-92

Zhang, YZ, Cheng H, Gould, KL, Golemis EA and Roder H 2003 Structure, stability and function of hDim1 investigated by NMR, circular dichroism and mutational analysis. Biochemistry $\mathbf{4 2}$ 9609-9618

Zhou HX, Rivas G and Minton AP 2008 Macromolecular crowding and confinement: biochemical, biophysical, and potential physiological consequences. Annu. Rev. Biophys. 37 375-397

MS received 16 July 2012; accepted 07 October 2012

Corresponding editor: DuRgadAs P KASBEKAR 